\title{
Metallic nanoshell for three-dimensional chemical mapping of low conductive materials with pulsed-voltage atom probe tomography
}

\author{
Vahid R Adineh $^{1}$, Ross K W Marceau ${ }^{2}$, Jing Fu ${ }^{1}$ \\ 1. Department of Mechanical Engineering, Monash University, Clayton, VIC 3800, Australia. \\ 2. Deakin University, Institute for Frontier Materials, Geelong, VIC 3216, Australia.
}

Although pulsed-laser atom probe tomography (APT) has been successfully applied to 3D imaging of insulated samples in recent years [1], it has been hypothesized that sufficient field evaporation can be achieved in pulsed-voltage APT given the presence of a thin, conductive layer on the specimen [2]. The aim of this study is to explore a new route of applying nanoscale metallic coating to facilitate pulsedvoltage APT imaging of insulated samples, which can then be applied to applications in various fields including biomaterials and cell biology.

We propose to employ a sub-10 nanometer metallic shell to "disguise" the insulated specimens and facilitate local field evaporation as suggested by the finite element electrostatic simulations (Fig. 1a). A schematic diagram of the proposed approach is presented in Fig. 1b. For the testing purpose, insulated samples such as resin used in electron microscopy with Au nanoparticles (NPs) embedded were retrieved by FIB lift-out and fabricated into needle shape with FIB annular milling (Fig. 1b). Physical vapor deposition (PVD) was tuned to coat a nanoscale metallic layer (Ag) on the insulated tips samples, which was confirmed with transmission electron microscopy (TEM) (Fig. 1b). The acquired mass spectrum from resin embedded Au NPs (Fig. 1c), is rich in the $1^{\text {st }}$ and $2^{\text {nd }}$ charge states of Au (197 Da and 98.5 Da, respectively) along with $1^{\text {st }}$ charge-state isotopes $\mathrm{Ag}(107$ and $109 \mathrm{Da})$. The rest of the peaks are likely pertaining to the resin matrix. Fig. 1d shows the acquired a 3D atom map by pulsed-voltage APT, from resin embedded $14 \mathrm{~nm}$ Au NPs with a $<5 \mathrm{~nm}$ Ag coating layer. Detailed atomic features have been observed including depleted and enriched regions likely due to an inhomogeneous distribution of $\mathrm{Au}$ atoms inside the NPs. The developed coating approach was further applied to investigate bacterial cells (A. baumannii) which were grown and dehydrated at prescribed time points. After lift-out, annular milling and acquisition by pulsed-voltage APT, an atom map was acquired from a cell envelope region (Fig. 1e), revealing complicated 3D structures that resemble network/fibril features [3].

In summary, applying an ultra-thin coating on the nanoscale APT tip samples facilitates pulsed-voltage field evaporation. The results showed that without the presence of a pulsed laser, $10^{6}-10^{7}$ ions could still be acquired from coated low conductivity materials [3]. The approach developed in this study is expected to provide a unique solution for exploring the architecture and chemistry of biological samples at the atomic scale.

References:

[1] Gordon LM, Joester D. Nature 469 (2011), p. 194-197.

[2] Kelly T F, et al. MRS bulletin 34 (2009), p. 744-750. 
[3] Adineh VR, et al. Nano Letters 374 (2016), p. 7113-7120.

[4] The authors acknowledge use of facilities within the Monash Centre for Electron Microscopy (MCEM) as well as Institute for Frontier Materials (IFM) - Deakin University. This study was funded by Monash University Interdisciplinary Research (IDR) Seed Fund and the Monash Centre for Atomically Thin Materials (MCATM) for the PhD top-up scholarship.

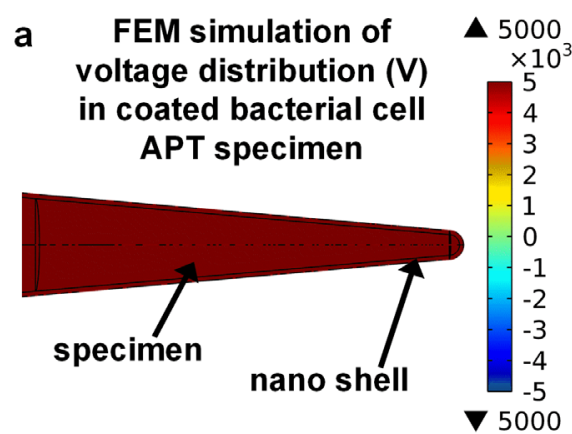

C

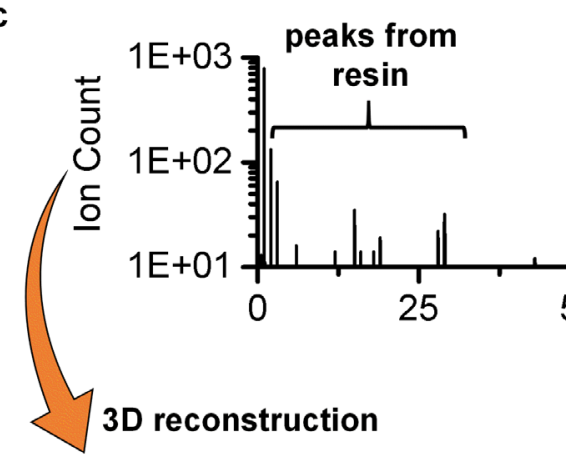

d

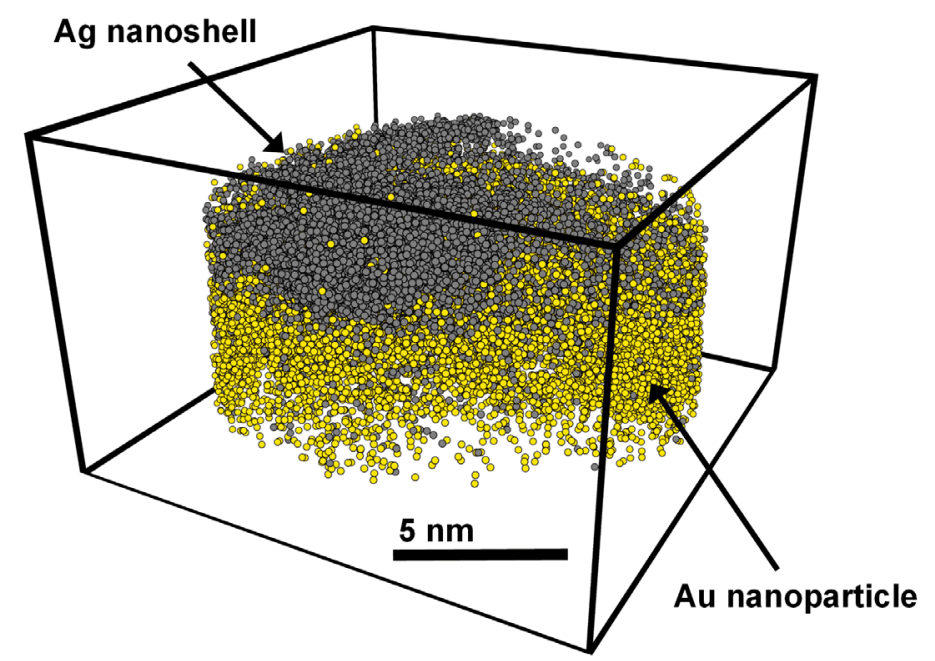

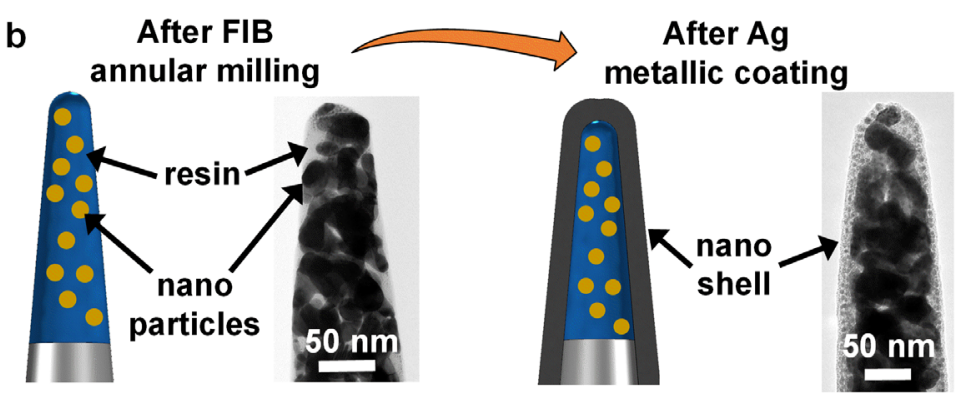

peaks from $\mathrm{Ag}$ nanoshell
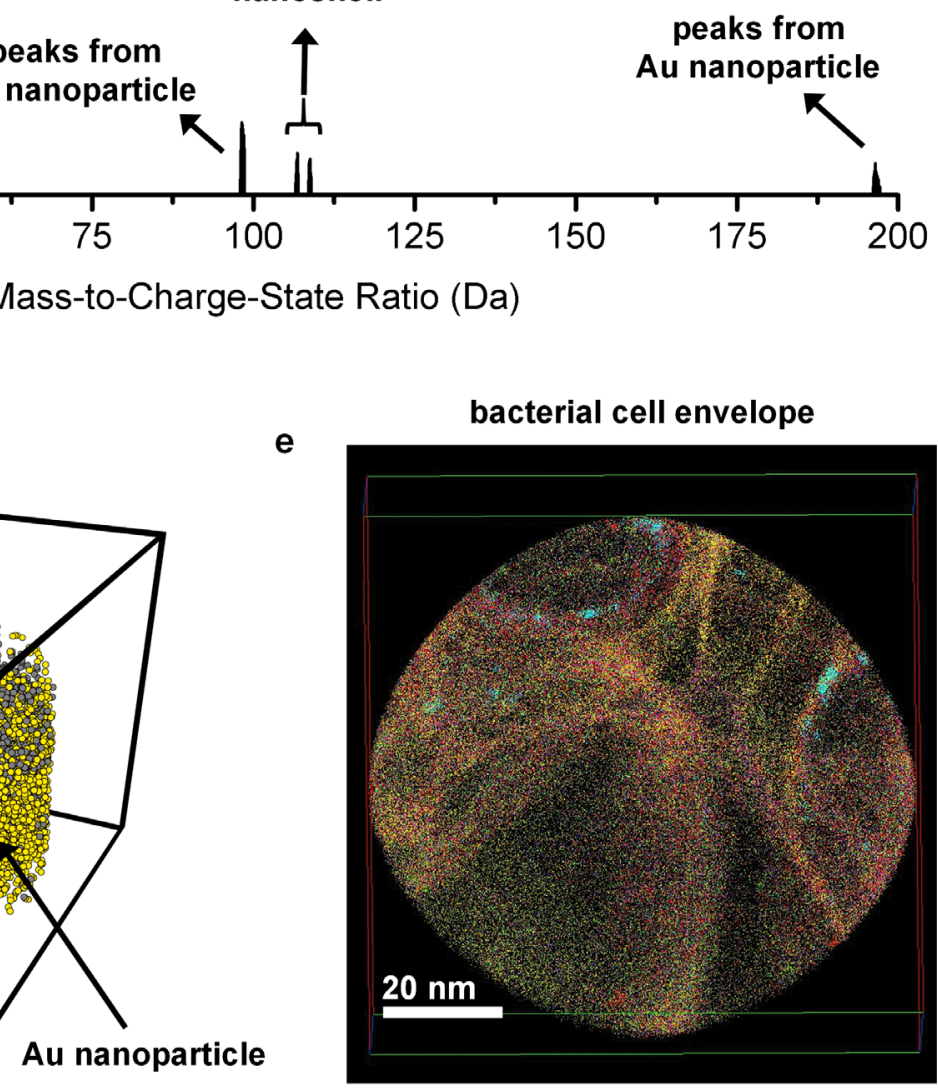

Figure 1. (a) Finite element simulation of voltage distribution in a bacterial cell APT specimen coated by an Ag nanoshell; (b) Schematic diagrams and TEM images showing FIB annular milling to produce sharp needle-form APT specimen of resin with Au NPs embedded, followed by nanoscale Ag coating; (c) acquired mass spectrum from resin embedded Au NPs; (d) 3D atom maps of Au NPs with partial Ag nanoshell; (e) a 3D atom map aquired from cell envelope region of a bacterial cell. 